\title{
Analysis of Acinetobacter baumannii resistance patterns in patients with chronic obstructive pulmonary disease (COPD) in terms of choice of effective empiric antibiotic therapy
}

\author{
Aneta Grochowalska', Maria Kozioł-Montewka², Anna Sobieszczańska ${ }^{3}$ \\ ${ }^{1}$ Laboratory of Microbiology, Masovian Specialistic Hospital in Radom, Radom, Poland \\ 2 Pope John II State School of Higher Education in Biała Podlaska, Biała Podlaska, Poland \\ ${ }^{3}$ Chair and Department of Jaw Orthopaedics, Medical University in Lublin, Lublin, Poland
}

Grochowalska A, Kozioł-Montewka M, Sobieszczańska A. Analysis of Acinetobacter baumannii resistance patterns in patients with chronic obstructive pulmonary disease (COPD) in terms of choice of effective empiric antibiotic therapy. Ann Agric Environ Med. $2017 ;$; 24(2): 307-311. https://doi.org/10.26444/aaem/74710

\begin{abstract}
Introduction. Multi-resistant Acinetobacter baumannii isolated from patients has become one of the most hazardous pathogens in health care settings. The aim of the study was to analyze pneumonia caused by Acinetobacter baumannii in patients hospitalized because of exacerbation of chronic obstructive pulmonary diseases (COPD), who were admitted to the Pulmonology Ward of the Masovian Specialistic Hospital in Radom (MSS). The incidence and drug sensitivity of these non-fermenting rods were evaluated, and compliance with antimicrobial procedure with the algorithm of the guidelines in applicable recommendations, was estimated. This should result in determining the local patterns of resistance and verifying therapeutic procedures in accordance with the assumptions of hospital antibiotic policy. In addition, the study examined the effectiveness of empiric and targeted therapy according to the clinical condition of the patient, and the eradication of A. baumannii, in comparison with the aggravating factors of the patient.

Materials and Method. The retrospective study included 90 patients with exacerbation of COPD whose etiological factor of infection was A. baumannii, hospitalized in the Department of Pulmonology (MSS) in 2012-2016.

Results. Studies were conducted on 90 patients with COPD exacerbation from which $A$. baumannii was isolated. Co-infections with other bacterial species among 41 patients were additionally noted. The majority of $A$. baumannii strains showed a high resistance ( $90 \%)$ to fluoroquinolones, ceftazidime, piperacillin/tazobactam. For strains causing a co-infection, drug resistance was successively $44-56 \%, 44 \%, 44 \%$. All of patients received empirical therapy. The most commonly used drug was amoxicillin with a clavulanic acid, often combined with fluoroquinolone. This type of therapy was effective among $10 \%$ of patients. The mortality in this group was determined at $29 \%$. Among $79 \%$ of patients with COPD, a targeted therapy was performed which proved to be effective in $58 \%$ of treated cases by susceptibility testing. The highest efficacy was observer after the use of colistin and carbapenems.

Conclusion. In the performed study, the infections caused by multi-resistant Acinetobacter baumannii, were observed in COPD, which should be taken into consideration in choosing empirical antibiotic therapy. Simultaneously, the local resistance patterns of multi-drug-resistant (MDR) Gram-negative strains co-infecting COPD should be considered in empirical treatment. Moreover, both additional clinical complication and co-infections contribute to a more severe course of diseases. In this study, the mortality percent exceeded $29 \%$.
\end{abstract}

\section{Key words}

COPD, antibiotics, Acinetobacter baumannii, multidrug-resistant strains, co-infection

\section{INTRODUCTION}

The WHO recognizes that chronic obstructive pulmonary disease (COPD) is of major public health importance [1], and refers to numerous professional groups, including farmers $[2,3,4]$. According to the WHO estimates in 2002, COPD was the fifth leading cause of death. Estimates show that in 2020 COPD will become the third leading cause of death worldwide [5]. In the referred group of patients, the exacerbations of COPD were often caused by bacterial infections. A large part of these bacteria represented the strains resistant to multiple drugs. Acinetobacter baumannii was identified as the etiological agent of infections in COPD

Addres for correspondence: Aneta Grochowalska, Laboratory of Microbiology, Masovian Specialistic Hospital in Radom, Radom, Poland

E-mail: aneta.grochowalska@op.pl

Received: 5 January 2017; accepted: 1 March 2017; first published on June 2017 patients, and became one of the most troublesome pathogens for health-care centres $[6,7]$. Data in the literature show that the problem of stress mortality rate is higher in patients with multidrug-resistant bacteria, compared to patients with other bacteria, and it is significantly associated with inappropriate initial antibiotic treatment [8].

At present, the dominant problem caused by A. baumannii infection is the presence of numerous multidrug-resistance (MDR) strains in the hospital environment, which are often insensitive to carbapenems and respond only to colistin (XDR) $[9,10]$. Hence, there is a growing problem of lack of drugs which would effectively combat these micro-organisms, because strains resistant even to colistin (PDR - pandrug resistance) have been isolated from clinical material. In the presented study, all $A$. baumannii strains were sensitive to colistin. In many cases, this polymyxin has been the only alternative treatment [11]. 
Another issue is the coinfection with other bacteria. The presence of tracheobronchial colonization, local and systemic immunosuppression, and frequent use of antibiotics are predisposing factors for complications in this group of patients. In this group of patients, the exacerbations of COPD were often caused by bacterial infections $[12,13]$. These infections are ranked as severe, with a high mortality rate [14].

Treatment. The patients were admitted to the Pulmonology Ward, depending on their pneumonia severity, the degree of respiratory failure, and the probability of mechanical ventilation usage. The decision for empiric antibiotic therapy should be based on the selection of drug correct for the clinical diagnosis. According to the prepared 'Guidelines on diagnosis, therapy and antibiotic prophylaxis of hospital infections' [15], the strategy should be taken into account of infection and risk factors etiology, I e: the overall condition of the patient, liver/renal failure or other health disorders, which are of great importance in the choice and dosage of antibiotics. After receiving the results from the laboratory of microbiology, in the case of unsuccessful empirical therapy, treatment should be targeted in accordance with antibiogram.

Therapeutic regiment. Exacerbations of COPD patients who require hospital admission are associated with significant in-patient mortality. The decision to administer antibiotics in exacerbation of COPD is multifactorial, and the most important considerations are severity of the COPD stage and patient performance status, clinical symptoms (increased dyspnea, sputum volume and sputum purulence), severity of current and previous exacerbations, comorbidity and current smoking.

Treating with antibiotics must include coverage for Haemophilus influenzae, Streptococcus pneumoniae and Moraxella catarrhalis. In patients with long-standing history of COPD and severe acute exacerbations of the disease, Gram-negative Enterobacteriaceae and non-fermenting rods are often isolated. As these patients tend to be hospitalized more frequently due to exacerbation episodes, the following microorganisms with acquired drug resistance can most often found: P. aeruginosa, A. baumannii, Burkholderia cepacia, and Stenotrophomonas maltophilia. Antibiotics, particularly macrolides and fluoroquinolones, when administered under suitable conditions, shorten the clinical course and prevent severe deterioration, possible complications. In these cases, the following antibiotics are recommended by therapeutic options, according to national guide. No risk factors age $<65$ years, $\mathrm{FEV}_{1}>50 \%,<2$ exacerbations per year without heart problems, therapy is recommended: cefuroxime $2 \times 500 \mathrm{mg}$ per os (po), or $3 \times 1.5 \mathrm{~g}$ intravenous (iv) or amoxycillin with clavulanic acid $3 \times 625 \mathrm{mg}$ po, or $2 \times 1,0 \mathrm{mg}$ po, excellent pharmacokinetics forms per os. With risk factors: age $>65$ years, $\mathrm{FEV}_{1}<50 \%, \geq 3$ exacerbations per year, with heart problems: ceftriaxone $1 \times 2 \mathrm{~g}$ iv levofloxacin or $1 \times 500 \mathrm{mg}$ po or iv. In case of risks with infection with $P$. aeruginosa: amoxycillin with clavulanic acid $(3 \times 625 \mathrm{mg}$ po) + ciprofloxacin $2 \times 400 \mathrm{mg}$ iv, ceftazidime $3 \times 1 \mathrm{~g}$ iv or levofloxacin $1 \times 500 \mathrm{mg}$ po or iv. Whereas, those more exposed to multiresistance should be given one of the following drugs: ceftazidime $3 \times 2 \mathrm{~g}$ iv, piperacillin-tazobactam $4 \times 4,5 \mathrm{~g}$ iv, imipenem $4 \times 0.5 \mathrm{~g}$ iv or meropenem $3 \times 1 \mathrm{~g}$ iv, cefepime 2-3×1-2 g iv. By default, the treatment should take from
3-5 days, in case it is longer, it will not bring any benefits, only an increased risk of undesirable symptoms [15].

\section{MATERIALS AND METHOD}

The subjects of microbiological and therapeutic analysis were patients admitted to hospital because exacerbation of COPD, whose etiological factor of infection was A. baumannii as the only microorganism isolated or concomitant infection. Microbiological analyses were performed on 90 strains of A. baumannii derived from 90 patients and 41 isolates of other species which additionally accompanied 41 patients, as a second causative agent of the infection. All the subjects were treated in MSS between 2012-2016. Test materials were those routinely collected for diagnostics - the secretions from the lower respiratory tract (sputum, BAL - bronchoalveolar washings) obtained from patients from pulmonology wards. For the analysis of A. baumannii strains, the principle of one strain $=$ one patient was applied.

From test materials received from the hospital wards, direct preparations stained with Gram method were made. For further diagnostics, the samples were qualified according to the amount of purulent secretions ( $>25$ leukocytes / neutrophils $<10$ epithelial cells in the visual field of the microscope. Microbiological diagnostics of COPD exacerbations considers quantitative culture secretions. To diagnose pneumonia, it was essential to assess the number and morphology of the bacteria in the direct preparation, and the fact of incubating from bronchial secretions collected with the BAL method in the quantitative plating $>10^{4} \mathrm{CFU}$ / $\mathrm{ml}$ of bacterial strains. In the case of sputum - colonies meeting the criterion of 'dominating' growth are diagnostic indications for pneumonia. The material was cultured in accordance with general principles of microbiological diagnostics. Standard bacteriological media (bioMerieux) was used. They included Columbia Agar with 5\% sheep blood, McConkey agar, Chapman Agar, Chocolate Agar selective for Haemophilus spp. Incubation was conducted in aerobic conditions in a $\mathrm{CO}_{2}$ enriched atmosphere at the temperature of $36^{\circ} \mathrm{C} \pm 1^{\circ} \mathrm{C}$, and bacterial growth was evaluated after 18-24-hours. Then, after determining the morphology of the cultured colony and microscopic observation, the species was identified by means of commercial kit including ID Cards and Vitek 2 Compact (bioMerieux).

Determination of antibiotic susceptibility of isolated from bacteria was made by three methods: disc diffusion (KirbyBauer), which uses discs (from Oxoid) method gradient diffusion E-test, and using an automatic camera Vitek 2 Compact, which uses cards with a set of antibiotics for Gram negative (bioMerieux).

Acinetobacter baumannii isolates insensitive to carbapenems were examined with EDTA test for MBL (metallo-beta-laktamases) presence, and with a test with boronic acid for KPC (Klebsiella pneumoniae carbapenemase).

The method of determining drug sensitivity, resistance mechanisms and their interpretation were performed as recommended by EUCAST, the National Reference Centre for Antimicrobial drug-sensitivity Testing, and according to the manufacturer's instructions.

Methodology. The study was retrospective. The research was conducted from 2012-2016 at the Pulmonology Ward of 
Masovian Specialistic Hospital in Radom (MSS). The study group included hospital patients who had Acinetobacter baumannii isolated from the lower respiratory tract, and having COPD symptoms of infectious exacerbation phase. Detailed data on: patient identification, the general condition of patients, risk factors for infection, diagnostic methods, diagnosed infections, and the method of treatment were taken from the patients' hospital records.

COPD symptoms of infectious exacerbation phase was diagnosed on the following basis: clinical manifestations observed in physical examination, laboratory results, microbial results, level of procalcitonin, leukocytosis or leukopenia, C-reactive protein concentration, a deterioration of gas exchange (of $\mathrm{PaO}_{2} / \mathrm{FiO}_{2}$ ratio), and radiological tests: chest X-ray and / or computer tomography.

Non-fermenting Gram-negative rods were mostly isolated from MSS patients diagnosed with exacerbation of chronic obstructive pulmonary disease (COPD).

\section{RESULTS}

At the time and in the wards mentioned above, 4,310 inoculations were performed in 3.535 patients with suspected pneumonia. The average number of days of hospitalization were 27 days, respectively.

There were 90 strains of Acinetobacter baumannii isolated from 90 patients with COPD symptoms of infectious exacerbation phase. The percentage of patients with this etiological agent represented $2.5 \%$ of all patients with suspected pneumonia. The test specimens were taken from 58 men and 32 women.

The aggravating factors among study group were analyzed. The average age was 69 years. In the clear majority of hospitalized patients $(n=71,79 \%)$, a respiratory failure was recorded on/during admission to hospital. 17 patients required artificial ventilation, including $9(10 \%)$ patients in the first days of stay in the clinic. Circulatory failure occurred among everyone $(n=90,100 \%)$ in the group, while renal failure among $5(17 \%)$ of patients. In addition, $28(31 \%)$ people suffered from diabetes, 10 (11\%) from obesity, 8 (9\%) from cancer, 12 (13\%) from alcohol addiction, and 51 (57\%) from nicotine addiction. Antibiotics treatment within 3 months before admission was confirmed by 50 (56\%) patients. All pulmonology patients $(n=90,100 \%)$ confirmed a previous stay in hospital within 90 days before the subsequent admission to MSS. In the study group, there were 90 cases diagnosed with COPD during the exacerbation phase of the infection in the Pulmonology Ward.

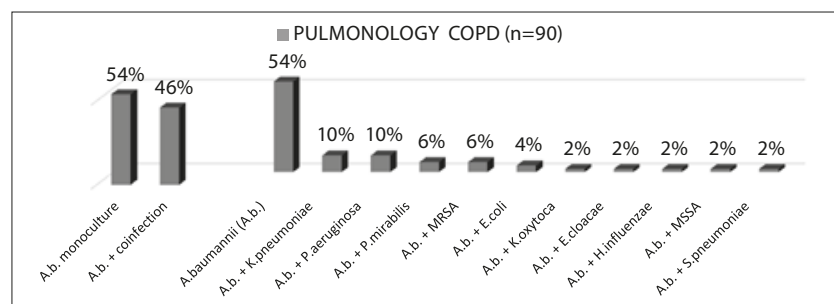

Figure 1. The proportion of COPD patients, with A. baumannii and associated pathogens

Abbreviations: A.b. - Acinetobacter baumannii

Of the 90 patients infected with A. baumannii, in 49 cases (54\%) A. baumannii was isolated in monoculture, while in the remaining 41 patients $(46 \%)$ with $P$. aeruginosa $(\mathrm{n}=9$, $10 \%), K$. pneumoniae $(\mathrm{n}=9,10 \%)$, P. mirabilis $(\mathrm{n}=5,6 \%)$, MRSA (methicillin-resistant Staphylococcus aureus, $\mathrm{n}=5$, $6 \%)$, E. coli (n=3,4\%), K. oxytoca, E. cloaceae, H. influenzae, S. pneumoniae, and MSSA (methicillin-sensitive Staphylococcus aureus, each=2,2\%). (Fig. 1).

Analysis of drug resistance of the cultured strains. In the group of 90 patients, A. baumannii strains isolated from pulmonology patients with exacerbated COPD the level of resistance to the recommended empirical antibiotic therapy, exceeded $85-90 \%$. The antibiotics included: piperacillin / tazobactam, ceftazidime, ciprofloxacin, levofloxacin, and other drugs: piperacillin, cefepime, tobramycin, trimethoprim / sulfamethoxazole.

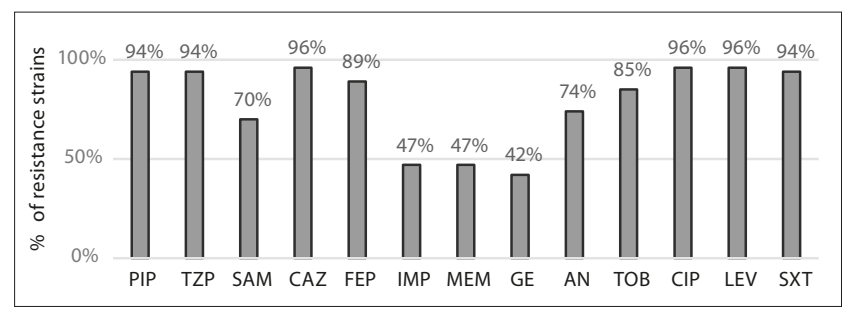

Figure 2. Drug-resistance of $A$. baumannii strains isolated from patients with COPD Abbreviations used: PIP - piperacillin, TZP - piperacillin / tazobactam, SAM ampicillin / sulbactam, CAZ - ceftazidime, FEP - cefepime, IMP - imipenem, MEM meropenem, GE - gentamicin, AN - amikacin, TOB - tobramycin, CIP - ciprofloxacin, LEV - levofloxacin, SXT - trimethoprim / sulfamethoxazole, $n$ - number of patients

Resistance to the aminoglycosides in the case of gentamicin was $42 \%$, whereas amikacin proved ineffective in $74 \%$ of cases. The level of strains resistance to carbapenems was $47 \%$ for both meropenem and imipenem. The degree of resistance to ampicillin with sulbactam reached $70 \%$ (Fig. 2).

It was also determined that COPD drug resistant microorganisms cause a co-infection with COPD patients to antibiotics, which should be used for empirical therapy.

The current study also aimed at determining the drug resistance of the remaining, other than A. baumannii strains, causing a co-infection.

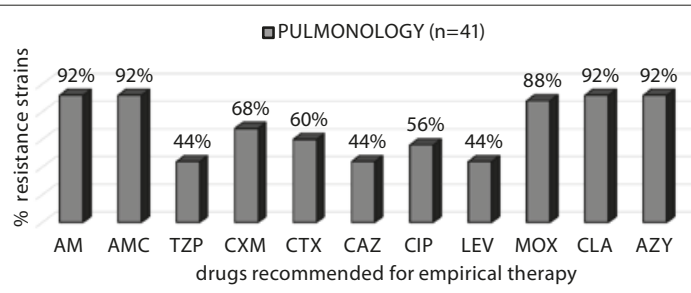

Figure 3. Drug-resistance of co-infection causing strains cultured from patients with COPD

Abbreviations: AM - ampicillin, AMC - amoxicillin / clavulanate, CXM - cefuroxime, CLA - clarithromycin, AZY - azithromycin, MOX - moxifloxacin, CTX - cefotaxime, LEV - levofloxacin, CAZ - ceftazidime, TZP - piperacillin / tazobactam, CIP ciprofloxacin, $\mathrm{n}$ - number of patients

The strains causing co-infection in pulmonology patients with COPD appeared to have various resistance to drugs recommended for empirical therapy. The lowest resistance percent (44\%) of tested strains were resistant to piperacillintazobactam, ceftazidime and levofloxacin. While the highest 92\% were resistant to: ampicillin, ampicillin with clavulanic acid, clarithromycin and azithromycin (Fig. 3) It should be also noticed, that the high percentage of resistance isolates 
concerns the hospitalized study group, which means, that these drugs may be used during empirical therapy in other groups not included in our study.

Analysis of the applied empirical treatment. All 90 patients received empirical treatment involving mostly amoxicillin with clavulanic acid in combination with ciprofloxacin $(\mathrm{n}=18)$, monotherapy amoxicillin-clavulanate $(n=15)$, ciprofloxacin $(\mathrm{n}=10)$, or fluoroquinolone in combination with amikacin $(n=10)$ or clindamycin $(n=10)$.

Table 1. The applied empirical treatment of patients with COPD in pulmonology ward

\begin{tabular}{lccc}
\hline $\begin{array}{l}\text { PULMONOLOGY } \\
\text { treatment }\end{array}$ & $\begin{array}{c}\mathrm{COPD}(\mathrm{n}=90) \\
\text { patients }\end{array}$ & $\begin{array}{c}\text { applied empirical } \\
\text { treatment }\end{array}$ & $\begin{array}{c}\mathrm{n}=90-\text { numer of } \\
\text { patients }\end{array}$ \\
\hline AMC & 15 & CIP & 10 \\
\hline AMC+CIP & 18 & CIP+CXM & 4 \\
\hline AMC+AN & 2 & CIP+CC & 10 \\
\hline AMC+CXM & 3 & CIP+AN+CXM & 2 \\
\hline AMC+MET & 2 & CXM & 3 \\
\hline AMC+CIP+AN & 4 & CTX+AN+CIP & 2 \\
\hline AN+CIP & 10 & $C C+M E T$ & 2 \\
\hline AN+CXM & 3 & & 2 \\
\hline
\end{tabular}

$\mathrm{AN}+\mathrm{CXM}$

3

Abbreviations: AMC - amoxicillin / clavulanate, CIP - ciprofloxacin, MET - metronidazole, CXM cefuroxime, CTX - cefotaxime, AN - amikacin, CC - clindamycin, $\mathrm{n}$ - number of patients.

For a better presentation of the empirical therapies used, cultured bacterial strains were examined also for their sensitivity to the drugs tested.

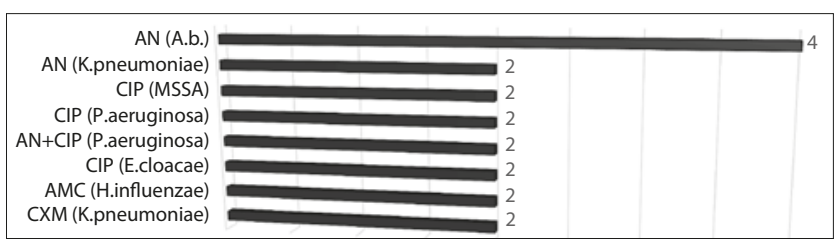

Figure 4. The number of strains sensitive to empiric therapy in patients with exacerbation of COPD

Abbreviations: A.b. - Acinetobacter baumannii, MSSA - Methicillin-susceptible Staphylococcus aureus, AN - amikacin, CIP - ciprofloxacin, AMC - amoxicillin / clavulanate, CXM - cefuroxime, $\mathrm{n}$ - number of patients

Empiric therapy complied with sensitive strains antibiogram in only 18 patients. The drugs used were: amoxicillin with clavulanic acid $(\mathrm{n}=2)-H$. influenzae, ciprofloxacin $(\mathrm{n}=6)-$ E. cloacae, $P$. aeruginosa, MSSA, cefuroxime $(\mathrm{n}=2)-K$. pneumoniae, amikacin $(\mathrm{n}=8)-$ K. pneumoniae and A. baumannii amikacin and ciprofloxacin - P. aeruginosa. 9 patients showed health improvement after application of the empirical therapy with ciprofloxacin $(n=4)$ or ciprofloxacin combined with amikacin $(n=5) .10$ patients died before the introduction of targeted therapy.

Analysis of applied targeted therapy. Due to the lack of satisfactory clinical improvement among patients, the remaining 71 received further targeted treatment. This additionally analyzed the applied targeted therapies, and also studied the obtained bacterial strains in terms of their sensitivity to the drugs used.

A group of 71 patients received selected targeted antibiotics. Meropenem as monotherapy $(n=19)$ and colistin $(n=18)$ were

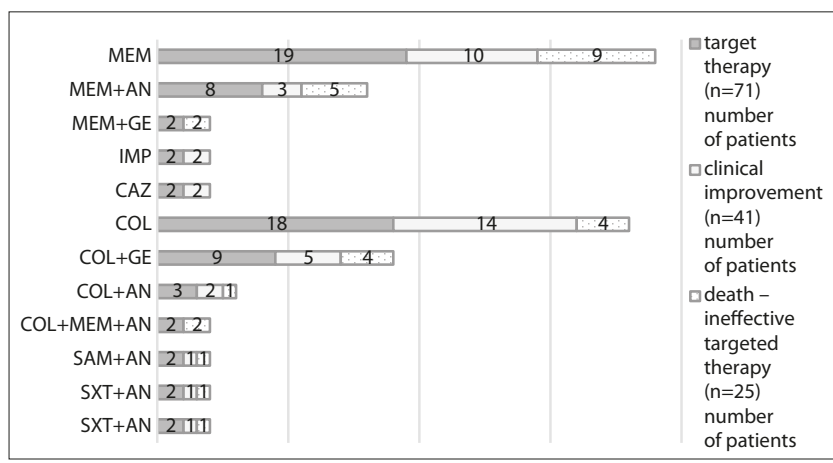

Figure 5. Applied targeted treatment and clinical improvement in patients with exacerbations of COPD

Abbreviations used: SXT - trimethoprim / sulfamethoxazole, GE - gentamicin, AN - amikacin, MEM - meropenem, IMP - imipenem, CAZ - ceftazidime, SAM ampicillin / sulbactam, COL - colistin

most often used in monotherapy, whereas a combination of colistin and gentamicin $(n=9)$ or amikacin $(n=5)$ were most often applied in combined therapy. From this group, 25 patients died, while another 5 were moved to other hospitals. In 41 patients, the treatment resulted in clinical improvement. The treatment involved the following antibiotics: meropenem $(n=10)$ or the combination of this drug with amikacin $(n=3)$, trimetropim / sulfamethoxazole $(n=1)$, or in association with amikacin $(\mathrm{n}=1)$, amikacin, ampicillin / sulbactam $(\mathrm{n}=1)$, colistin $(n=14)$, or its combination with gentamicin $(n=5)$ or amikacin $(n=2)$, imipenem $(n=2)$ and ceftazidime $(n=2)$.

\section{DISCUSSION}

The growing incidence of $A$. baumannii in clinical specimens of the hospital patients hospitalized is also a global problem. The infection under study caused by these non-fermenting rods, were caused mainly by multi-resistant strains. The results of the current study s are consistent with reports in the literature $[16,17]$.

Our research led to the conclusion that co-infection with the most common cause of Gram-negative bacilli. Generally, Klebsiella pneumoniae, Pseudomonas aeruginosa. Isolation of $A$. baumannii infections species have been associated with prolonged hospitalization and severe airway obstruction [18].

It has been reported that from the whole group of 90 COPD patients, 25 (29\%) of them died. The deaths were mainly caused by severe conditions originating from primary diseases and lack of success during antibiotic therapy. However, in 9 cases, the pathogen was eliminated, which means that in two subsequent microbiological tests, A. baumannii was not isolated. 17 patients were additionally treated with respiratory ventilators.

Before the therapy, the patients should be tested for the most likely etiologic agent of infection. When choosing the right antibiotic and its dosage, certain risk factors affecting the patient's condition, such as liver or kidney failure and other disorders, should be taken into consideration.

In the current study, analysis showed that when using the recommended empirical therapy drug sensitivity of the chance to hit against a co-infection-causing microorganism would be more effective compared to the treatment used. It was noted that the recommendations for empiric reatment were not always followed. Often misused the treatment by amoxicillin clavulanic acid is not active against pathogenic 
bacteria in hospitals, which are typically more resistant to multiple drugs. It should be emphasized that improper use of empiric therapy leads to the selection of resistant organisms in the hospital environment, and disturbing the physiological flora of the patient. The destruction of microflora of the patient often results in infection by $C$. difficile $[19,20]$.

It is noteworthy that most empirical therapy showed no activity against the microorganisms isolated from patients. Microbiological tests confirmed that A. baumannii strains selected from the lower respiratory tract were difficult to predict in terms of drug sensitivity, thus the treatment should be carried out according to susceptibility testing. For this reason, a targeted therapy against A. baumannii most often used colistin or carbapenems (imipenem, meropenem). This procedure corresponds to data in the literature $[21,22]$.

In the presented study group, despite the applied targeted antibiotic therapy, some patients died due to severe conditions caused by primary diseases which decreased the patients' immunity.

All strains tested showed sensitivity to colistin which belongs to the polymyxin antibiotic of last resort against infections caused by strains of $A$. baumannii resistant to many drugs. From the literature, it is known that there are already numerous strains resistant to colistin. For this reason, we should use this medicine rationally $[23,24]$.

\section{CONCLUSIONS}

1) Determining the etiology of bacterial exacerbation of COPD is difficult and depends on several varying factors affecting the severity of the condition of the patient, including a history of antibiotic therapy over the last three months.

2) Certain diagnostic and therapeutic difficulties may result from the fact that some pathogenic species colonizing the airways of COPD patients do not cause any aggravation of the disease.

3) Infection symptoms caused by A. baumannii are similar to the symptoms caused by other Gram negative bacilli.

4) The exacerbations for COPD empirical therapy caused by $A$. baumannii should be based on recommended guidelines, and adjusted according to local data collected by monitoring the microbes' susceptibility.

5) The most common cause of treatment failure in this group of patients are: serious general condition of the patient, reduced immunity, heart failure and respiratory failure.

\section{REFERENCES}

1. Lopez AD, Shibuya K, Rao C, Mathers CD, Hansell AL, Held LS, Schmid V, Buist S. Chronic obstructive pulmonary disease: current burden and future projections. Eur Respir J. 2006; 27(2): 397-412.

2. Oliynyk O, Doroshenko M, Slifirczyk A. Evaluation of calcium homeostasis in patients treated in Ternopil University Hospital for chronic obstructive pulmonary disease. Health Problems of Civilization. 2016; 10(4): 60-64.

3. Stoleski S, Minov J, Karadzinska-Bislimovska J, Mijakoski D. Chronic obstructive pulmonary disease in never-smoking dairy farmers. Open Respir Med J. 2015; 31;9: 59-66.
4. Szczyrek M, Krawczyk P, Milanowski J, Jastrzębska I, Zwolak A, Daniluk J. Chronic obstructive pulmonary disease in farmers and agricultural workers - an overview. Ann Agric Environ Med. 2011; 18(2): 310-313.

5. Donaldson GC, Wedzicha JA. Review series, COPD exacerbations 1: Epidemiology. Thorax 2006; 61: 164-168.

6. Lin SH, Kuo PH, Hsueh PR, Yang PC, Kuo SH. Sputum bacteriology in hospitalized patients with acute exacerbation of chronic obstructive pulmonary disease in Taiwan with an emphasis on Klebsiella pneumoniae and Pseudomonas aeruginosa. Respirology. 2007; 12(1): 81-7.

7. Noweta K, Frankowska M, Grzelewska-Rzymowska. Exacerbations of chronic obstructive pulmonary disease and the role of sputum bacteriological examination. Pneumonol Alergol Pol. 2006; 74(4): 396-402.

8. Nseir S, Ader F. Prevalence and outcome of severe chronic obstructive pulmonary disease exacerbations caused by multidrug-resistant bacteria. Curr Opin Pulm Med. 2008; 14(2): 95-100.

9. Tripathi PC, Gajbhiye SR, Agrawal GN. Clinical and antimicrobial profile of Acinetobacter spp.: An emerging nosocomial superbug. Adv Biomed Res. 2014; 9;3: 13.

10. Mastoraki A, Douka E, Kriaras I, Stravopodis G, Saroglou G, Geroulanos S. Preventing strategy of multidrug-resistant Acinetobacter baumannii susceptible only to colistin in cardiac surgical intensive care units. Eur J Cardiothorac Surg. 2008; 33(6): 1086-90.

11. Tan CH, Li J, Nation RL. Activity of colistin against heteroresistant Acinetobacter baumannii and emergence of resistance in an in vitro pharmacokinetic/pharmacodynamic model. Antimicrob Agents Chemother. 2007; 51(9): 3413-5.

12. Ye D, Shan J, Huang Y, Li J, Li C, Liu X, et al. A gloves-associated outbreak of imipenem-resistant Acinetobacter baumannii in an intensive care unit in Guangdong, China. BMC Infect Dis. 2015; 11;15: 179.

13. Shete VB, Ghadage DP, Muley VA, Bhore AV.Lung India. Multi-drug resistant Acinetobacter ventilator-associated pneumonia. 2010; 27(4): 217-20.

14. Townsend J, Park AN, Gander R, Orr K, Arocha D, Zhang S, Greenberg DE. Acinetobacter infections and outcomes at an academic medical center: a disease of long-term care. Open Forum Infect Dis. 2015; 12;2(1): ofv023.

15. Hryniewicz W, Ozorowski T, et al. Rekomendacje diagnostyki, terapii i profilaktyki antybiotykowej zakażeń w szpitalu. Narodowy Program Ochrony Antybiotyków. 2015. www.antybiotyki.edu.pl

16. Odewale G, Adefioye OJ, Ojo J, Adewumi FA, Olowe OA. Multidrug Resistance of Acinetobacter Baumannii in Ladoke Akintola University Teaching Hospital, Osogbo, Nigeria. Eur J Microbiol Immunol (Bp). 2016; 29;6(3): 238-243.

17. Osei Sekyere J. Current State of Resistance to Antibiotics of Last-Resort in South Africa: A Review from a Public Health Perspective. Front Public Health. 2016; 30;4: 209.

18. Nakou A, Papaparaskevas J, Diamantea F, Skarmoutsou N, Polychronopoulos V, Tsakris A. A prospective study on bacterial and atypical etiology of acute exacerbation in chronic obstructive pulmonary disease. Future Microbiol. 2014; 9(11): 1251-60.

19. Keeley AJ, Beeching NJ, Stott KE, Roberts P, Watson AJ, Beadsworth MB. Clostridium difficile: A healthcare-associated infection of unknown significance in adults in sub-Saharan Africa. Malawi Med J. 2016; 28(2): 66-69.

20. Rodriguez C, Taminiau B, Korsak N, Avesani V, Van Broeck J, Brach P, Delmée M, Daube G. Longitudinal survey of Clostridium difficile presence and gut microbiota composition in a Belgian nursing home. BMC Microbiol. 2016; 1;16(1): 229.

21. Li XJ, Li Q, Si LY, Yuan QY. Bacteriological differences between COPD exacerbation and community-acquired pneumonia. Respir Care. 2011; 56(11): 1818-24.

22. Rice LB. Challenges in identifying new antimicrobial agents effective for treating infections with Acinetobacter baumannii and Pseudomonas aeruginosa. Clin Infect Dis. 2006; 1;43 Suppl 2: S100-5.

23. Mu X, Wang N, Li X, Shi K, Zhou Z, Yu Y, et al. The Effect of Colistin Resistance-Associated Mutations on the Fitness of Acinetobacter baumannii. Front Microbiol. 2016; 1;7: 1715.

24. Rossi F, Girardello R, Cury AP, Gioia TS, Almeida JN Jr, Duarte AJ. Emergence of colistin resistance in the largest university hospital complex of São Paulo, Brazil, over five years. Braz J Infect Dis. 2016; 7. pii: S1413-8670(16)30442-1. 